\title{
How Does Chronic Venous Disease Progress from the First Symptoms to the Advanced Stages? A Review
}

Nicos Labropoulos

Received: January 17, 2019 / Published online: February 13, 2019

(C) The Author(s) 2019

\section{ABSTRACT}

Risk factors for the development of progression chronic venous disease (CVD) and varicose veins are widespread and include advanced age, excess body weight, sedentary lifestyles and occupations, family history, and pregnancy. Varicose veins and CVD are associated with venous hypertension, venous reflux, dysfunctional venous valves, and vein wall inflammation, though the precise etiologies are unclear. Once venous pathology develops, it can progress through a vicious cycle of inflammation and leukocyte recruitment that leads to further deterioration of vein walls and valves, increased hypertension, and release of additional proinflammatory mediators. Early treatment of symptomatic varicose veins and CVD as well as lifestyle changes can help break the inflammatory cycle and alleviate symptoms. Physicians and patients should be aware of the risk factors for CVD, the treatments and measures available to slow disease progression, and the serious

Enhanced Digital Features To view enhanced digital features for this article go to https://doi.org/10.6084/ m9.figshare.7599161.

N. Labropoulos $(\bowtie)$

Department of Surgery, Stony Brook University

Medical Center, Stony Brook, NY, USA

e-mail: nlabrop@yahoo.com consequences of allowing the disease to progress unchecked.

Funding: Servier (France).

Keywords: Cardiology; Chronic venous disease; Chronic venous insufficiency; Venous hypertension; Venous inflammation; Venous reflux

\section{INTRODUCTION}

Risk factors for the development of chronic venous disease (CVD) and its progression are widespread [1-3]. Some factors can be mitigated through lifestyle changes to increase physical activity, stop smoking, and reduce excess bodyweight, but others are unmodifiable. For example, people with a family history of varicose veins or CVD are predisposed to developing these same conditions, aging is a strong and unavoidable risk factor, and the prevalence of CVD tends to be higher in women, especially if they have been pregnant. While having children is a choice, it is unlikely that many women would avoid pregnancy for the sole reason of reducing the risk of developing varicose veins. In addition, reducing excess bodyweight and preventing its return are difficult for many overweight or obese individuals. Given the inevitability of developing CVD in many individuals, it is critical to understand how CVD progresses and how this progression can be slowed or prevented. 
This article is based on previously conducted studies and does not contain any studies with human participants or animals performed bythe author.

\section{CLINICAL PROGRESSION OF CVD}

CVD progression is defined as a change in clinical signs that indicate an increase in CEAP class, as a change in symptoms, or changes in both $[4,5]$. Progression of CVD is highly variable and proceeds along different pathways in different patients, though some patterns are evident [5-7]. In addition, the early signs and/or symptoms of CVD cover a wide spectrum of presentation. For example, some individuals with superficial venous reflux may be symptomatic but without varicose veins, while others with varicose veins may or may not have venous symptoms [6].

Examples of progression have been investigated in CVD patients waiting for or delaying elective vein surgery [6]. Among 116 limbs in 90 patients, reflux detected by duplex ultrasound was the predominant pathology and was present in $97.4 \%$ of the limbs. At a second ultrasound scan 1-43 months later, disease had progressed in 31 limbs (26.7\%), of which 14 (12.1\%) had a new site of reflux and $17(14.7 \%)$ had an extension of pre-existing reflux. Thirteen limbs (11.2\%) had progressed to a new clinical stage: seven went from $\mathrm{C} 2$ to $\mathrm{C} 3$, four from $\mathrm{C} 3$ to $\mathrm{C} 4$, and two from $\mathrm{C} 4$ to $\mathrm{C} 6$; however, new reflux was detected in only seven of these limbs.

A clinical case illustrates an example of progression with symptom worsening but no change in venous reflux. A female patient with two children and a family history of varicose veins (mother) presented with heaviness, itching, and varicosities in the right lower limb and reticular veins, but without symptoms in the left leg. Duplex ultrasound demonstrated reflux in the great saphenous vein (GSV) from the junction to the mid-thigh with a varicose tributary extending to the lower calf. The patient agreed only to therapy with elastic compression stockings. She returned 34 months later complaining of swelling, pain on standing, heaviness, and itching in the right leg, though the left leg remained asymptomatic. A second duplex scan showed no changes from the previous examination, suggesting that the symptom worsening could be due to microcirculation deterioration. No other factors were implicated as the patient had no other comorbidities and no changes in either physical activity or bodyweight.

\section{MECHANISMS OF DISEASE PROGRESSION}

Chronic venous hypertension and dilatation lead to a number of pathophysiologic changes in the vein wall and surrounding tissues $[8,9]$. Altered shear stress and initial inflammatory processes at the vein wall lead to endothelial cell activation, which increases the permeability of the endothelium. In addition, abnormal hemodynamics allows multiple other factors to come into play, such as hypoxia, dysregulated apoptosis, and extracellular matrix changes, all of which can influence varicose vein development at the cellular level [10]. Following red blood cell extravasation through the damaged endothelium and vein wall and red blood cell breakdown, fibrin and hemosiderin concentrations increase in the tissues and activate mast cells and macrophages, which contribute to further endothelial cell activation and inflammation. Activated endothelium also triggers adhesion and migration of leukocytes through the vein wall, where they release TGF- $\beta_{1}$ and proinflammatory cytokines, all of which stimulate collagen synthesis by fibroblasts, increase vascular wall thickening and remodeling, and potentiate inflammatory cascades.

One of the critical events that begins this vicious cycle of inflammation and tissue destruction is the infiltration of leukocytes through the venous endothelium into the vein wall and surrounding tissues. This occurs at endothelial cell junctions through a process known as diapedesis in which it appears that leukocyte signaling directs the rearrangement of cell-cell junctions such as adherens junctions formed by vascular endothelial cadherins and tight junctions formed by claudins [11]. 
Chronic inflammation alone can have deleterious consequences on venous flow. Arterial and venous flow patterns in legs of patients with C4-C6 CVD were found to be similar to those in patients with leg inflammation caused by cellulitis [12]. Healthy volunteers $(n=20)$ exhibited normal phasic blood flow in all GSVs, calf perforator veins, and popliteal veins. Among patients with C2 CVD $(n=21)$, abnormal continuous flow indicating venous valve dysfunction was found in only two patients. In contrast, between 19 and 24 patients with C4-C6 CVD $(n=29)$ exhibited abnormal continuous venous flow with or without hyperemic (excess) flow in at least one of these veins, and all patients with cellulitis $(n=12)$ had abnormal flow patterns in at least two of the veins. In a relative measure of inflammation, patients with C2 disease were approximately four times more likely than healthy controls to have enlarged lymph nodes, and patients with C4-C6 disease were approximately 13 times more likely. These results suggest that inflammation can lead to abnormal venous flow and that inflammation severity parallels the severity of CVD.

The microcirculation also becomes increasingly impaired in CVD. Vasomotor activity in the skin microvasculature of CVD patients tends to decrease with increasing CVD class, and the amplitude of vasomotor cycles is significantly higher in $\mathrm{C} 4-\mathrm{C} 6$ patients than in C1-C3 patients [13]. As CVD progresses in severity, the microvasculature network in ulcerated skin becomes very dense and highly disorganized. Such pathologic deterioration of the microcirculation is consistent with the classical signs and symptoms of C4-C6 CVD.

In CVD progression, the risk factors that lead to chronic venous hypertension and dilatation, and the consequent inflammation, also create a vicious circle of pathologies that worsen abnormal venous flow, further increase local venous pressure, damage venous valves, and potentiate inflammation (Fig. 1) [14]. Local inflammation brought on by leukocyte adhesion and infiltration leads to structural alterations in the walls and valves of veins, which increase venous reflux, capillary hypertension, leakage, and edema, all of which contribute to inflammatory responses in the surrounding tissues and skin, and can eventually lead to ulceration.

However, a second case history illustrates that severe GSV reflux alone, without varicose veins or obstruction, can lead to symptoms. The case in question was a 39-year-old mother of three children who presented with leg tenderness and local pain that was most severe at day's end and was relieved by leg elevation. She had no varicose veins but severe GSV reflux lasting over $5640 \mathrm{~ms}$, which extended from the preterminal valve to the upper calf and into some first- to third-order tributaries. Small saphenous and deep veins were normal with no reflux or obstruction. The patient was diagnosed with subclinical segmental CVD (CO s). Such a patient at early-stage CVD warrants treatment to relieve symptoms and prevent disease progression. Standard conservative treatment could begin with compression stockings and a vasoactive drug such as MPFF (micronized purified flavonoid fraction, e.g., Daflon(R)) along with follow-up to determine whether the treatment is acceptable and effective. Although effective for symptom relief, compression stockings have not been shown to slow progression or prevent recurrence of varicose veins [15]. Thus, the addition of MPFF, which has been shown to effectively reduce the signs, symptoms, and inflammation related to CVD, may help slow progression. Invasive endovenous treatment at this stage is controversial, and no guidelines exist for its use in such patients.

\section{THE NATURAL HISTORY OF CVD PROGRESSION}

Epidemiologic evidence from large cohorts shows that CVD progresses at a substantial rate, portending marked increases in patient burdens in coming decades as the population ages and life expectancies become longer. In the Framingham Study of 3822 adults, the annual incidence of varicose veins was $2.6 \%$ in women and $1.9 \%$ in men [2]. Incidences of varicose veins and CVD reached $14 \%$ in the Bonn Vein Study after 6.6 years of follow-up [16]. Progression in 


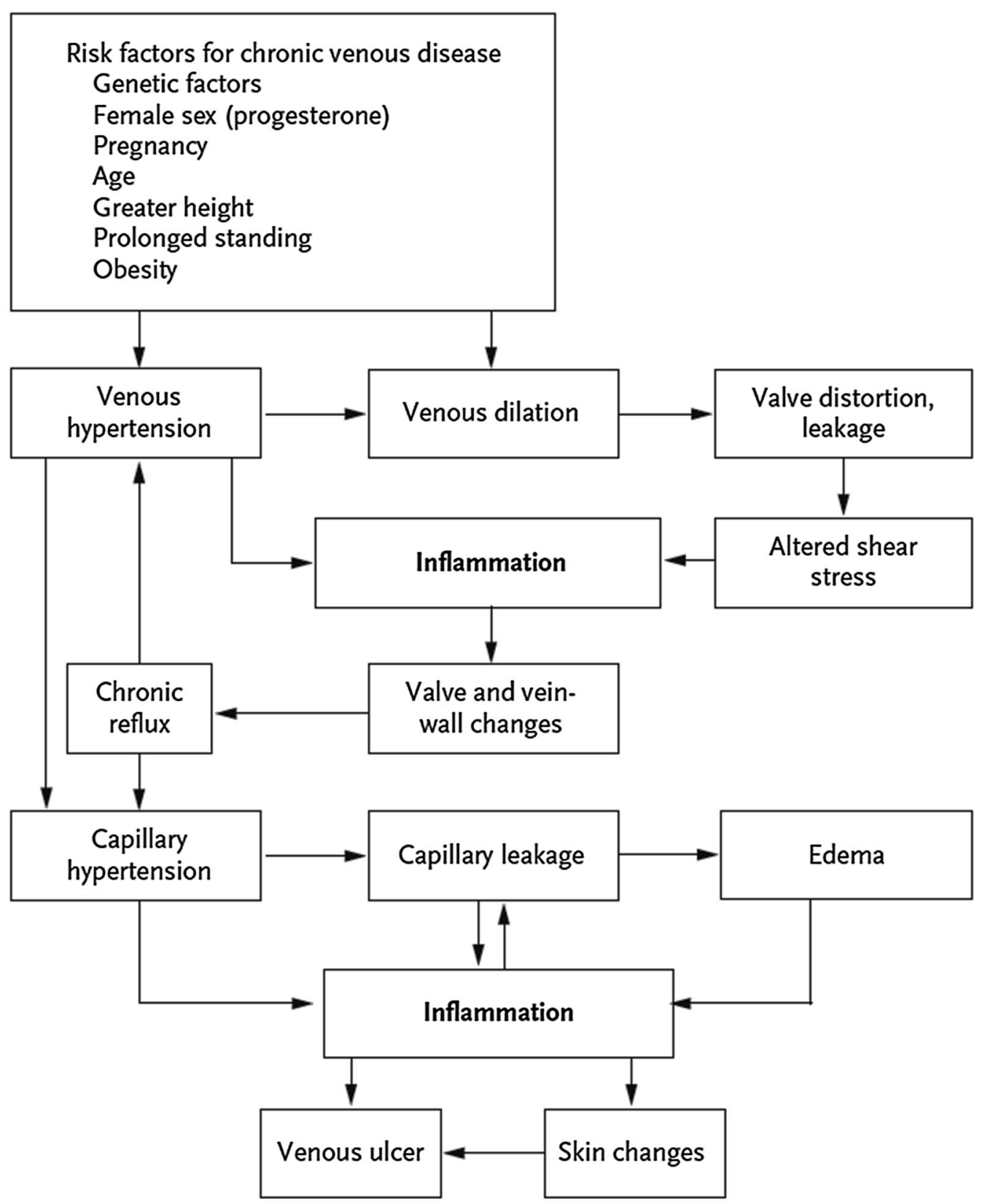

Fig. 1 CVD development and progression are due to vicious cycles of venous hypertension, inflammation, capillary damage, and edema. From New England Journal of Medicine, Bergan JJ, Schmid-Schonbein GW, Coleridge

this study was much higher in C2 patients with saphenous varicose veins, $31 \%$ of whom progressed to C3-C6 over the follow-up period. The main risk factors for progression were advanced age, high BMI, and symptoms of swelling sensation in patients with varicose veins. In the
Smith PD. et al., Chronic Venous Disease, 355., 488-498. Copyright $^{(}$(2006) Massachusetts Medical Society. Reprinted with permission from Massachusetts Medical Society [14]

Edinburgh Vein Study, $57.8 \%$ of 334 CVD patients had progressed to more severe varicose veins or CVD, or developed new varicose veins, after a mean follow-up period of 13.4 years, with an annual progression rate of 5\% [5]. Almost $1 / 3$ of patients with varicose veins 
developed skin changes, putting them at risk of ulceration. The lifestyle and clinical factors most strongly related to progression were advanced age, family history of varicose veins, history of deep vein thrombosis, high BMI, and venous reflux. In particular, reflux in the small saphenous vein coupled with deep reflux, was likely to be associated with a higher risk of progression. That venous reflux, particularly superficial combined with deep venous reflux, was associated with greater progression suggests that duplex scanning could be a useful prognostic tool to identify patients at risk for rapid progression.

High progression rates have also been confirmed in other studies [5-7, 16-19]. However, additional studies are needed to determine the risk of progression to venous ulcers, which greatly impact patient QoL and healthcare resources, and to define which patients may benefit from early vein interventions to prevent progression. Clearly, CVD progression cannot be ignored, and interventions are needed to prevent deterioration, benefit patients, and reduce healthcare costs.

\section{RECOMMENDATIONS}

There is ample published evidence to show that, when patients are managed appropriately, conservative and interventional treatments for CVD can significantly improve disease-specific and generic health-related QoL, relieve a wide range of troublesome lower limb symptoms, delay and prevent the onset of complications of CVD, and provide a highly cost-effective use of healthcare resources $[20,21]$. Though prevention is not currently possible, patients with risk factors should be monitored for signs of earlystage disease and treated appropriately to manage disease progression. Patients should be informed of the risk factors for CVD and its consequences and should be encouraged to reduce risk where possible. In all applicable cases, lifestyle changes are warranted to increase physical activity, stop smoking, and reduce excess bodyweight. In patients with subclinical segmental disease, compression stockings with or without MPFF are recommended for symptomatic relief [15]. However, because compliance can be low with compression therapy, these patients should be followed up to determine whether this therapy is acceptable or alternative measures are needed. Therapy with venoactive drugs may also be recommended, many of which have demonstrated efficacy for the signs and symptoms of CVD and can slow progression [21]. In patients with advanced CVD, such as those presenting with edema, skin changes, or ulceration, aggressive interventions are necessary and physicians should consider procedures to treat reflux and obstruction, while compression therapy and venoactive drugs have been shown to promote ulcer healing.

\section{CONCLUSIONS}

There is no prophylaxis for varicose veins, though some risk factors are modifiable. Without appropriate treatment, CVD will progress in most cases because of a cycle of chronic inflammation that leads to further deterioration in venous flow, venous hypertension, and edema. Early treatment of symptomatic varicose veins or CVD, however, can improve symptoms and signs as well as health-related QoL and can slow progression. However, varicose veins removed through surgical or endovenous methods are very likely to recur despite treatment. Preventative programs may help to inform patients about the risk factors for CVD, how they can reduce risk, the early symptoms, and the serious consequences of progression. To slow CVD progression, patients in general should control their weight, increase mobility and physical activity, wear compression, if applicable, and use venoactive pharmacologic treatments demonstrated to be effective for the signs and symptoms of CVD.

\section{ACKNOWLEDGEMENTS}

Funding. This supplement has been sponsored by Servier. The article processing charges and open access fee were funded by Servier 
(France). The author had full access to all of the data for this study and takes complete responsibility for the integrity of the data and accuracy of the data analysis.

Medical Writing. The medical writing services were provided by Dr. Kurt Liittschwager (4Clinics, France) and were funded by Servier (France).

Authorship. Nicos Labropoulos meets the International Committee of Medical Journal Editors (ICMJE) criteria for authorship for this article, takes responsibility for the integrity of the work as a whole, and has approved this version for publication.

Prior Presentation. This article and all of the articles in this supplement are based on the International satellite symposium at European Venous Forum, June 29th, Athens.

Disclosures. Nicos Labropoulos received honoraria from Servier for the symposium lecture and in the past for serving on the Servier speaker bureau. The symposium was funded by an educational grant from Servier.

Compliance with Ethics Guidelines. This article is based on previously conducted studies and does not contain any studies with human participants or animals performed by the author.

Open Access. This article is distributed under the terms of the Creative Commons Attribution-NonCommercial 4.0 International License (http://creativecommons.org/licenses/ by-nc/4.0/), which permits any noncommercial use, distribution, and reproduction in any medium, provided you give appropriate credit to the original author(s) and the source, provide a link to the Creative Commons license, and indicate if changes were made.

\section{REFERENCES}

1. Beebe-Dimmer JL, Pfeifer JR, Engle JS, Schottenfeld D. The epidemiology of chronic venous insufficiency and varicose veins. Ann Epidemiol. 2005;15(3):175-84 (Epub 2005/02/23).

2. Brand FN, Dannenberg AL, Abbott RD, Kannel WB. The epidemiology of varicose veins: the Framingham Study. Am J Prev Med. 1988;4(2):96-101 (Epub 1988/03/01).

3. Robertson L, Evans C, Fowkes FG. Epidemiology of chronic venous disease. Phlebology. 2008;23(3):103-11 (Epub 2008/05/10).

4. Eklof B, Rutherford RB, Bergan JJ, Carpentier PH, Gloviczki P, Kistner RL, et al. Revision of the CEAP classification for chronic venous disorders: consensus statement. J Vasc Surg. 2004;40(6):1248-52 (Epub 2004/12/29).

5. Lee AJ, Robertson LA, Boghossian SM, Allan PL, Ruckley CV, Fowkes FG, et al. Progression of varicose veins and chronic venous insufficiency in the general population in the Edinburgh Vein Study. J Vasc Surg Venous Lymphat Disord. 2015;3(1):18-26 (Epub 2015/01/01).

6. Labropoulos N, Leon L, Kwon S, Tassiopoulos A, Gonzalez-Fajardo JA, Kang SS, et al. Study of the venous reflux progression. J Vasc Surg. 2005;41(2):291-5 (Epub 2005/03/16).

7. Pannier F, Rabe E. Progression in venous pathology. Phlebology. 2015;30(1 Suppl):95-7 (Epub 2015/03/ 03).

8. Bergan J. Molecular mechanisms in chronic venous insufficiency. Ann Vasc Surg. 2007;21(3):260-6 (Epub 2007/05/09).

9. Castro-Ferreira R, Cardoso R, Leite-Moreira A, Mansilha A. The role of endothelial dysfunction and inflammation in chronic venous disease. Ann Vasc Surg. 2018;46:380-93 (Epub 2017/07/10).

10. Jacobs BN, Andraska EA, Obi AT, Wakefield TW. Pathophysiology of varicose veins. J Vasc Surg Venous Lymphat Disord. 2017;5(3):460-7 (Epub 2017/04/17).

11. Dejana E. Endothelial cell-cell junctions: happy together. Nat Rev Mol Cell Biol. 2004;5(4):261-70 (Epub 2004/04/09).

12. Labropoulos N, Leder DM, Kang SS, Mansour MA, Baker WH. Inflammation parallels severity of chronic venous insufficiency. Phlebology. 2003;18(2):78-82.

13. Labropoulos N, Wierks C, Golts E, Volteas SK, Leon $\mathrm{M}$, Volteas N, et al. Microcirculatory changes parallel the clinical deterioration of chronic venous insufficiency. Phlebology. 2004;19(2):81-6 (Epub June 1, 2004). 
14. Bergan JJ, Schmid-Schonbein GW, Smith PD, Nicolaides AN, Boisseau MR, Eklof B. Chronic venous disease. N Engl J Med. 2006;355(5):488-98 (Epub 2006/08/04).

15. Palfreyman SJ, Michaels JA. A systematic review of compression hosiery for uncomplicated varicose veins. Phlebology. 2009;24(Suppl 1):13-33 (Epub 2009/05/16).

16. Rabe E, Pannier F, Ko A, Berboth G, Hoffmann B, Hertel S. Incidence of varicose veins, chronic venous insufficiency, and progression of the disease in the bonn vein study II. J Vasc Surg. 2010;51(3):791.

17. Engelhorn CA, Manetti R, Baviera MM, Bombonato GM, Lonardoni M, Cassou MF, et al. Progression of reflux patterns in saphenous veins of women with chronic venous valvular insufficiency. Phlebology. 2012;27(1):25-32 (Epub 2011/09/10).

18. Kostas TI, Ioannou CV, Drygiannakis I, Georgakarakos E, Kounos C, Tsetis D, et al. Chronic venous disease progression and modification of predisposing factors. J Vasc Surg. 2010;51(4):900-7 (Epub 2010/03/30).

19. Labropoulos N, Gasparis AP, Pefanis D, Leon LR Jr, Tassiopoulos AK. Secondary chronic venous disease progresses faster than primary. J Vasc Surg. 2009;49(3):704-10 (Epub 2009/03/10).

20. Berridge D, Bradbury AW, Davies AH, Gohel M, Nyamekye I, Renton S, et al. Recommendations for the referral and treatment of patients with lower limb chronic venous insufficiency (including varicose veins). Phlebology. 2011;26(3):91-3 (Epub 2011/04/08).

21. Nicolaides A, Kakkos S, Baekgaard N, Comerota A, de Maesenner M, Eklof B, et al. Management of chronic venous disorders of the lower limbs. Guidelines according to scientific evidence. Part I. Int Angiol. 2018;37(3):181-254 (Epub 2018/06/ 07). 